\title{
EStudios EGerenciales
}

Journal of Management and Economics for Iberoamerica

Artículo de investigación

\section{Evaluación del desempeño de la cadena de suministro sostenible enfocada en procesos}

Andrey Vinajera-Zamora *

Profesor-Investigador, Facultad de Ingeniería Mecánica e Industrial, Universidad Central "Marta Abreu” de Las Villas, Villa Clara, Cuba. andreyvz@uclv.edu.cu

Fernando Marrero-Delgado

Profesor-Investigador, Facultad de Ingeniería Mecánica e Industrial, Universidad Central “Marta Abreu” de Las Villas, Villa Clara, Cuba. fmarrero@uclv.edu.cu

Roberto Cespón-Castro

Profesor-Investigador, Facultad de Ingeniería Mecánica e Industrial, Universidad Central “Marta Abreu” de Las Villas, Villa Clara, Cuba. rcespon@uclv.edu.cu

\section{Resumen}

El presente artículo tiene como objetivo desarrollar una herramienta metodológica para evaluar el desempeño de la cadena de suministro sostenible mediante un índice integral. La metodología consta de cinco pasos, enfocada en procesos, y utiliza métodos como análisis de ciclo de vida y jerarquías analíticas o de Saaty (con base en múltiples criterios). Los criterios considerados fueron productividad, servicio al cliente, valor agregado, ecoeficiencia y rentabilidad. La metodología y el índice integral fueron aplicados a una cadena de suministro de contadores de energía eléctrica. Se consideraron las dimensiones económica, ambiental y social. Como resultado esencial, se obtuvo un mejoramiento de la sostenibilidad de la cadena estudiada, lo que demostró la novedad y la factibilidad de aplicación de esta herramienta.

Palabras clave: desempeño; cadena de suministro sostenible; multicriterio.

\section{Evaluation of sustainable supply chain performance focused on processes}

Abstract

This article aims to develop a methodological tool to evaluate the performance of the sustainable supply chain by an overall index. The methodology consists of five steps, focused on processes, and using methods such as life cycle analysis and analytical or Saaty hierarchies (based on multiple criteria). The criteria considered were productivity, customer service, added value, eco-efficiency and profitability. The methodology and the overall index were applied to a supply chain of electrical energy meters. The economic, environmental, and social dimensions were considered. As an essential result, an improvement in the sustainability of the chain studied was obtained, which demonstrated the novelty and the feasibility of applying this tool.

Keywords: performance; sustainable supply chain; multicriteria.

\section{Avaliação de desempenho da cadeia de abastecimento sustentável focada em processos}

Resumo

Este artigo tem como objetivo desenvolver uma ferramenta metodológica para avaliar o desempenho da cadeia de abastecimento sustentável por meio de um índice integral. A metodologia consiste em cinco etapas, com foco em processos, e utiliza métodos como análise do ciclo de vida e hierarquias analíticas ou de Saaty (baseadas em múltiplos critérios). Os critérios considerados foram produtividade, atendimento, valor agregado, ecoeficiência e rentabilidade. A metodologia e o índice integral foram aplicados a uma cadeia de abastecimento de medidores de eletricidade. As dimensões econômica, ambiental e social foram consideradas. Como resultado essencial, obteve-se uma melhoria na sustentabilidade da cadeia estudada, o que demonstrou a novidade e a viabilidade de aplicação desta ferramenta.

Palavras-chave: desempenho; cadeia de abastecimento sustentável; multicritério.

*Autor para dirigir correspondencia.

Clasificación JEL: D24; L25.

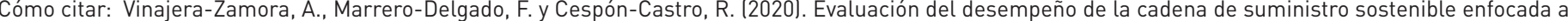
procesos. Estudios Gerenciales, 36(156), 325-336. https://doi.org/10.18046/j.estger.2020.156.3699

DOI: https://doi.org/10.18046/j.estger.2020.156.3699

Recibido: 6-sep-2019

Aceptado: 2-jul-2020

Publicado: 28-sep-2020 


\section{Introducción}

El incremento de la complejidad de las cadenas de suministros (CS) lleva a que los investigadores reconozcan la necesidad de medir y monitorear su funcionamiento, para evaluar el desempeño organizacional, gestionar efectivamente las actividades y ser más competitivo (AvelarSosa, García-Alcaraz y Maldonado-Macías, 2019; Hemalatha, Rao, Rambabu y Venkatasubbaiah, 2017; Sánchez-Flores, Ojeda-Benítez, Cruz-Sotelo y Navarro-González, 2020). Esta complejidad es consistente con la cantidad de actores presentes, por lo que ha sido un aspecto debatido en la literatura (Narimissa, Kangarani-Farahani y Molla-AlizadehZavardehi, 2020; Mohaghar, 2014). También se reconocen las necesidades de medir el desempeño de la CS (Ahi y Searcy, 2015), de poseer capacidades ágiles como condiciones necesarias para maximizar el desempeño de la cadena de suministro sostenible (Gey, Yusuf, Menhat, Abubakar y Ogbuke, 2020) y de administrar la relación entre los participantes y sus recursos (Icarte-Ahumada, 2016). En la actualidad, el desempeño de las CS se considera un factor clave de análisis para el mejoramiento de los procesos de dichas cadenas (Kumar, Kumar, Rao y Veeramalla, 2019).

La mejora continua de las CS requiere de la integración efectiva de todos los elementos que la componen (Parmenter, 2015). Este enfoque de gestión hace necesario reducir el riesgo y el impacto ambiental negativo a los niveles fijados (Dey y Cheffi, 2013), lo que acentúa la utilidad de considerar varios criterios para evaluar y mejorar el desempeño de la CS, mediante métricas integradas en un indicador o índice general (Anand y Grover, 2015).

Las expectativas del siglo XXI han cambiado y se observa un énfasis en las prácticas limpias y la dimensión social, unido a la conservación del tradicional criterio económico en el desempeño de una CS, con el nombre de cadenas de suministro sostenibles (CSS) (Chacón-Vargas y MorenoMantilla, 2016; Chin, Tat y Sulaiman, 2015). La sostenibilidad en el desempeño de la CS (Katiyar, Meena, Barua, Tibrewala y Kumar, 2018] ha ganado interés entre académicos y empresarios gracias a la consideración de aspectos sociales y ambientales (Mani, Gunasekaran y Delgado, 2018). Esto requiere de mecanismos robustos de medición, necesarios para transitar hacia una economía más limpia (Acquaye, Ibn, Genovese, Afrifa, Yamoah y Oppon, 2017). Las métricas empleadas en esta evaluación están dirigidas a los actores clave, al contexto de sostenibilidad y a sus características.

Varios investigadores han realizado importantes aportes utilizando técnicas con enfoque difuso, debido a la incertidumbre de la información para evaluar el desempeño, sin mostrar un indicador integral. A su vez, estos indicadores están compuestos por criterios con un enfoque funcional o departamental de la CS (Acquaye et al. 2017; Chin et al., 2015; Jakhar, 2015; Sopadang, Wichaisri y Banomyong, 2017; Tong, 2017; Uygun y Dede, 2016; Wang, 2012; Zhang, 2017) y no por procesos, con la excepción de Tsai y Hung (2009). Estos autores, aunque analizaron el desempeño de CS mediante criterios con enfoque en procesos internos, solo consideraron los eslabones de abastecimiento y manufactura, por medio de criterios de las dimensiones económica y ambiental. También fueron utilizados métodos basados en múltiples criterios, como el de jerarquías analíticas o de Saaty (AHP, por su sigla en inglés), pero sin proponer un indicador integral, y los criterios utilizados fueron funcionales.

Los autores mencionados en el tema de la evaluación del desempeño de CSS han realizado aportes importantes, entre los que resaltan el empleo de diferentes herramientas matemáticas, que incluyen métodos basados en múltiples criterios, el tratamiento de hasta dos dimensiones del desarrollo sostenible y algunas incursiones en el enfoque en procesos de las CS. Todos combinan métodos de investigación cuantitativos y cualitativos, lo cual es acertado en estos estudios. Sin embargo, dejan una brecha en la investigación en cuanto a la definición de un indicador integral para evaluar toda la CS con enfoque en procesos, que considere las tres dimensiones (económica, ambiental y social) del desarrollo sostenible.

Es opinión de los autores del presente artículo que, si bien es importante la evaluación de procesos y eslabones de una CS, también es necesario evaluarla de manera integral. Esta afirmación se justifica por la presencia de las denominadas relaciones de conflicto en la toma de decisiones, en las que las mejoras en algún proceso pueden deteriorar el funcionamiento de otros y de la CS como un todo. Esto se acentúa cuando se consideran diferentes dimensiones de la sostenibilidad, por lo que las medidas que se apliquen deben mejorar el desempeño de toda la CS. Por esa razón, el objetivo general del presente artículo fue desarrollar una herramienta metodológica para evaluar el desempeño de CSS mediante un indicador integral, compuesto por indicadores parciales enfocados en procesos, que considere las tres dimensiones del desarrollo sostenible y utilice métodos basados en múltiples criterios.

Para demostrar la factibilidad y bondades de la herramienta metodológica desarrollada, se tomó como estudio de caso la CS cubana de contadores de energía eléctrica. Esta CS utilizaba un enfoque en procesos en la gestión, pero basaba la toma de decisiones logísticas en los tradicionales criterios económicos y carecía de una herramienta que incluyera criterios ambientales y sociales; esta situación problemática originó la investigación. Para facilitar la lectura y comprensión, el artículo fue estructurado en las siguientes secciones: introducción, marco teórico, metodología, análisis de los resultados y conclusiones.

\section{Marco teórico}

Durante las últimas dos décadas, la gestión de la CS se ha convertido en un tema importante de investigación en la literatura de negocios. También se ha demostrado que las CS bien gestionadas pueden proporcionar ventajas competitivas para las organizaciones, las regiones y los países. Se reconoce que para lograr ese objetivo se necesita ser productivo ISun, Xu y Li, 2020; Elgazzar, Tipi y Jones, 2019; Kumar, Shivashankar y Kadadevaramath, 2017; Vinajera-Zamora, Marrero-Delgado, Coello-Machado y Glistau, 2015), agregar valor en cada uno de los procesos (Kaido y Katsuhito, 2020; 
Schenk, Thuronyi y Cui 2016), ser rentable (Tripathi y Talukder, 2020; Dossou y Nachidi, 2017; Mathivathanan, Govindan y Haq, 2017; Moharamkhani, Amiri y Mina, 2017; Montero, Schmalenberg, Quirós y Doluschitz, 2018; Tortorella, Giglio y Limon-Romero, 2018), mejorar continuamente (Egiguren y Elordi 2011), lograr el enfoque en el cliente (Tyagi, Kumar y Kumar, 2017) y gestionar la CS por procesos (Tundys y Fernando, 2020; Miri-Lavassani, 2018).

La CS vista desde una perspectiva económica, ambiental y social ha llamado la atención de los investigadores, dado el aumento de la conciencia social respecto a estas dimensiones. Al mismo tiempo, se ha producido un cambio en las filosofías tradicionales de gestión (Mediavilla, Errasti y Domingo, 2011), ya que, además de velar por los objetivos económicos, también se debe cumplir con objetivos sociales y ambientales (Mishra, Gunasekaran, Papadopoulos y Hazen, 2017; Narimissa et al., 2020; Saeed y Kersten, 2017; Tyagi, Kumar y Kumar, 2018). A este nuevo enfoque se le denomina gestión de la CSS (Ala-Harja y Helo, 2016; Feitó-Cespón, Cespón-Castro y Rubio-Rodríguez, 2016). Algunos autores abordan este concepto desde posiciones tecnológicas, políticas, operacionales y culturales, que pueden variar entre países y áreas geográficas (Gopal y Thakkar, 2015; Guan, Cheng y Ye, 2010).
Para evaluar y mejorar el desempeño de la CSS, se emplean métricas que permiten medirla (Sánchez-Flores et al., 2020) por procesos (Tundys y Fernando, 2020), departamentos, eslabones de la cadena, proyectos, grupos e individuos. Así, al realizar una búsqueda en la literatura usando el término desempeño de la CSS Isustainable supply chain performance), se identificó un total de 62 investigaciones. El 31\% (19) realiza una revisión de la literatura o identifica criterios que sirven de ayuda para la evaluación de su desempeño, sin mostrar cómo evaluarla. Del $69 \%$ restante, 22 (36\%) no muestran contribuciones sobre su evaluación y solo en las 21 investigaciones que aparecen en la figura 1 se ofrecen formas de medir el desempeño de las CSS.

La figura 1 contiene cuatro vertientes: al método empleado para el cálculo o evaluación, b) enfoque de dicha evaluación (global ${ }^{1}$ o detallado ${ }^{2}$ ), c) integración de los criterios en un indicador o índice y d) propuesta de la escala de evaluación.

En la figura 1 (a) se observa la tendencia que empleará la lógica difusa con tratamientos diferentes. Zhang (2017) y Tong (2017) utilizaron operadores; y Tyagi, Kumar y Kumar (2015), la técnica difusa (Fuzzy Technique for Order of Preference by Similarity to Ideal Solution [TOPSIS]), para encontrar la mejor alternativa de mejorar el desempeño de CSS. Mohaghar (2014) usó un método de programación difusa
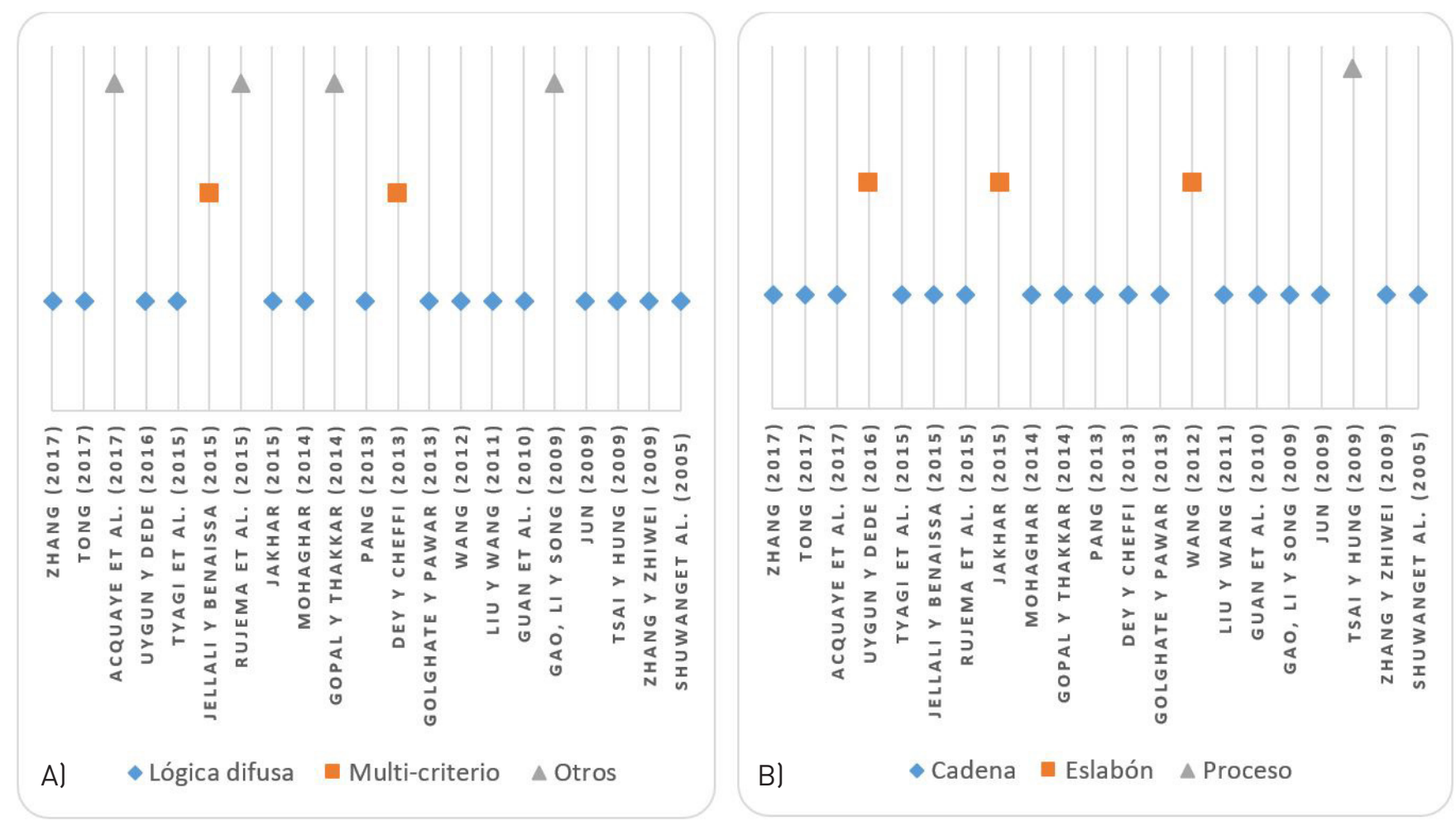

Figura 1. Contribuciones dirigidas a la evaluación de las CSS. la - método utilizado; b - enfoque de la investigación; c - integra criterios en un indicador; d - propone escala de evaluación).

Fuente: elaboración propia.

\footnotetext{
1 La evaluación del desempeño de CSS se realizó a partir de criterios aplicados a macroprocesos, eslabones o a la CS en su conjunto.

${ }^{2}$ La evaluación se realizó a partir de criterios aplicados a procesos internos de la CS.
} 


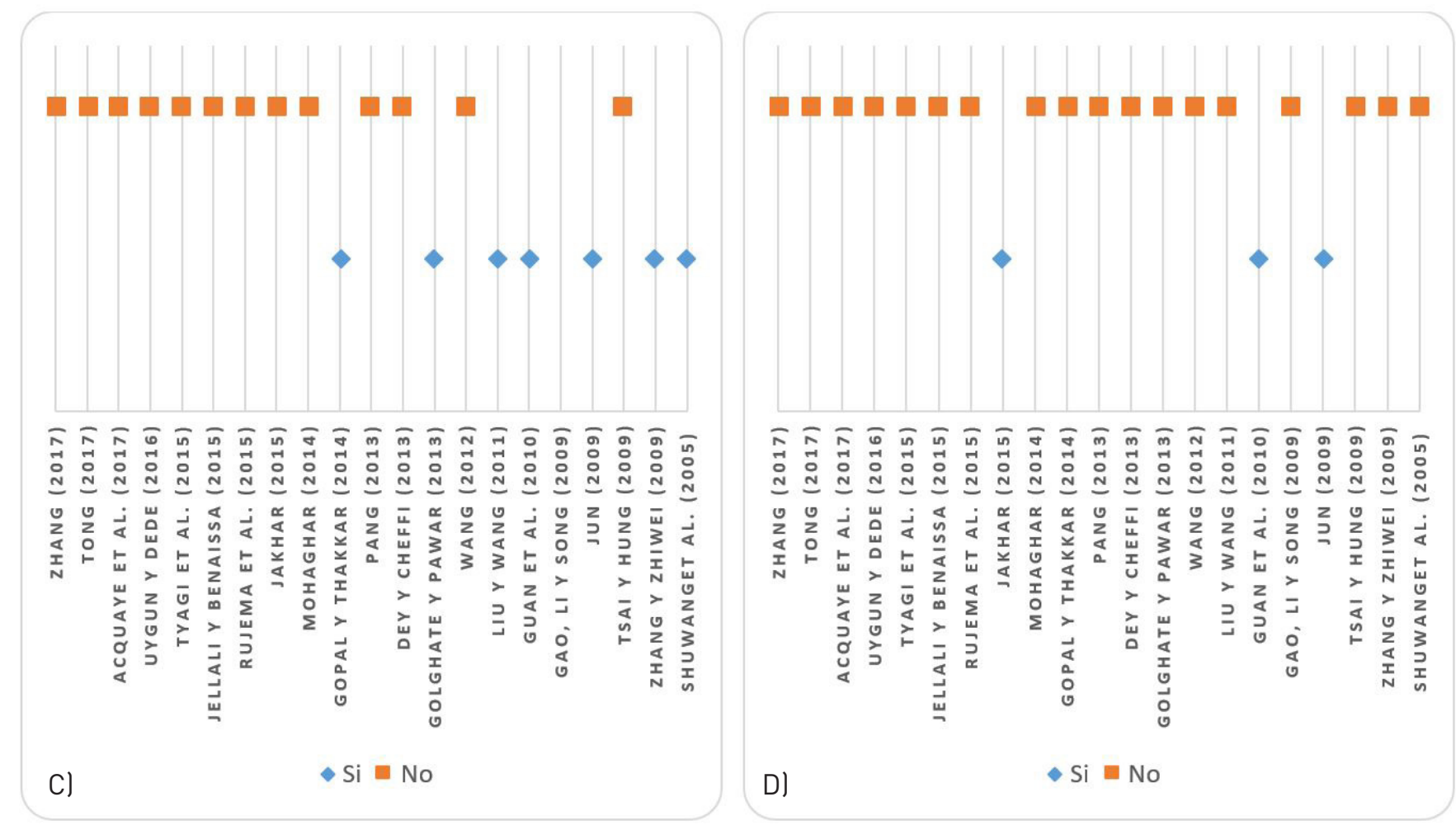

Figura 1. Contribuciones dirigidas a la evaluación de las CSS. la - método utilizado; b - enfoque de la investigación; c - integra criterios en un indicador; d - propone escala de evaluación). (Continuación)

Fuente: elaboración propia.

de preferencia logarítmica; y Pang (2013), el exhaustivo difuso multinivel para crear el sistema de indicadores. En cambio, Uygun y Dede (2016) utilizaron TOPSIS para evaluar el desempeño de CSS; Jakhar (2015), un modelo de programación lineal con múltiples objetivos para obtener la mejor estrategia; y Wang (2012), un método difuso de evaluación exhaustiva. Los criterios empleados en estas investigaciones fueron funcionales. Ello significa que no se realizó previamente un estudio de los procesos len diferentes niveles) que componen la CS, para, en función de ellos, definir los criterios de evaluación. Una herramienta que ha demostrado un elevado potencial para el enfoque en procesos es el modelo de referencia para las operaciones de la CS (SCOR, por sus siglas en inglés) (Jamehshooran, Shaharoun, Haron, 2015; Moharamkhani et al., 2017; Sellitto, Pereira, Borchardt, da Silva, Viegas, 2015).

Tsai y Hung (2009) proponen un modelo de programación metadifusa; Golghate y Pawar (2013), un análisis de lógica difusa; y Liu y Wang (2011) y Guan et al. (2010) aplicaron un método difuso de evaluación exhaustiva para determinar el desempeño de una CSS. Jun (2009) utilizó un método de evaluación por síntesis de los niveles difusos multietapa para evaluar el desempeño en grupos de CSS; Zhang y Zhiwei (2009), un modelo difuso de evaluación exhaustiva; y Shuwang, Lei, Zhifeng y Guangfu (2005), una evaluación difusa de dos capas.

Se identificaron dos investigaciones que emplearon métodos basados en múltiples criterios. Jellali y Benaissa (2015) utilizaron estos métodos para obtener una clasificación de las mejores prácticas en el mejoramiento del desempeño de la CSS, y Dey y Cheffi (2013) utilizaron el AHP para determinar los criterios más importantes en el desempeño de las CSS analizadas. Los criterios empleados en estas investigaciones también fueron funcionales.

En la figura 1(a), aparecen además investigaciones que emplearon otros métodos. Acquaye et al. (2017) utilizaron un modelo cuantitativo para medir la sostenibilidad de la CS desde perspectivas ambientales. Rujema, Richu y Aquilars (2015) usaron herramientas estadísticas lestadística descriptiva e inferencial y pruebas de correlación) para analizar el efecto de las estrategias de compra en el desempeño de la CSS; Gopal y Thakkar (2015), la técnica de polígono en la evaluación del análisis del ciclo de vida (ACV); y Gao, Li y Song (2009), un algoritmo de conversión de miembros con igual objetivo.

En la figura 1(b), se observa que solo un trabajo presenta un enfoque en procesos. Es el caso de Tsai y Hung (2009), que, como se comentó, utilizaron una perspectiva de análisis de los procesos de abastecimiento y manufactura, y los criterios empleados analizaron solo las dimensiones económicas y ambientales. En la figura 1 (c), se muestra la integración de criterios en un indicador en el que se evidencian las investigaciones de Gopal y Thakkar (2015), Golghate y Pawar (2013), Liu y Wang (2011), Guan et al; (2010), Jun (2009), Zhang y Zhiwei (2009) y Shuwang et al; (2005). En estas, se emplearon criterios funcionales. La figura 1(d) muestra las investigaciones que proponen una escala de evaluación, entre estas se destacan las de Jakhar (2015), 
Guan et al. (2010) y Jun (2009), aunque también con enfoque funcional.

A pesar de que los diferentes métodos para evaluar el desempeño de la CS, ya descritos, no son en su mayoría excluyentes, la totalidad de los autores consultados prefieren el empleo de un método en particular. Otro análisis realizado se refiere a los criterios analizados en estas investigaciones. En la figura 2 se muestran los criterios abordados en cada una de las 21 investigaciones antes mencionadas.

A partir de los aportes mostrados en la figura 2, en la presente investigación fueron seleccionados los criterios de rentabilidad, servicio al cliente, productividad o eficiencia, valor agregado e impacto ambiental. El valor agregado se incorpora como consecuencia de la aplicación del método propuesto y es explicado más adelante en el punto correspondiente a los resultados. Los criterios planteados se corresponden con el estudio de caso que se analiza en el presente artículo, pues el método propuesto es flexible al cambio de criterios, independiente de la CS que se analice.

El análisis de las investigaciones contenidas en las figuras 1 y 2 corrobora que el objetivo que se plantea en el presente trabajo resulta de interés en la literatura especializada y en la práctica empresarial.

Por esa razón, al utilizar métodos basados en múltiples criterios, para obtener un indicador integral e indicadores parciales con un enfoque en procesos para evaluar la CSS, y con base en los criterios señalados en el párrafo anterior, se afirma que la herramienta metodológica propuesta permite las siguientes acciones:
- Calcular el índice integral de desempeño de la cadena de suministro sostenible (SCPI) bajo un enfoque detallado, es decir, aplicar los criterios a los procesos.

- Emplear criterios desde las dimensiones económicas (valor agregado y rentabilidad), operacionales (productividad), ambientales (ecoeficiencia, utilizando el ACV) y sociales (servicio al cliente) de la sostenibilidad.

- Exponer una escala para la evaluación de la CS a partir del SCPI calculado.

\section{Metodología}

El método propuesto está compuesto por cinco pasos de trabajo que son explicados a continuación:

- Paso 1. Seleccionar criterios para calcular el SCPI. Se identificaron los posibles criterios que podrían servir para calcular el desempeño de la CSS. Primero, se selecciona un grupo de expertos en el tema, que pueda, de forma rápida y eficiente, identificarlos y seleccionarlos. Luego, se realiza una búsqueda en la literatura que permite la identificación de los posibles criterios para considerar en la evaluación. Una vez definidos, se seleccionan los que se ajustan a las características del objeto de estudio. Para ello, se emplean grupos de expertos apoyados por métodos de clasificación. Los criterios seleccionados para la evaluación analizan las dimensiones económicas, ambientales y sociales.

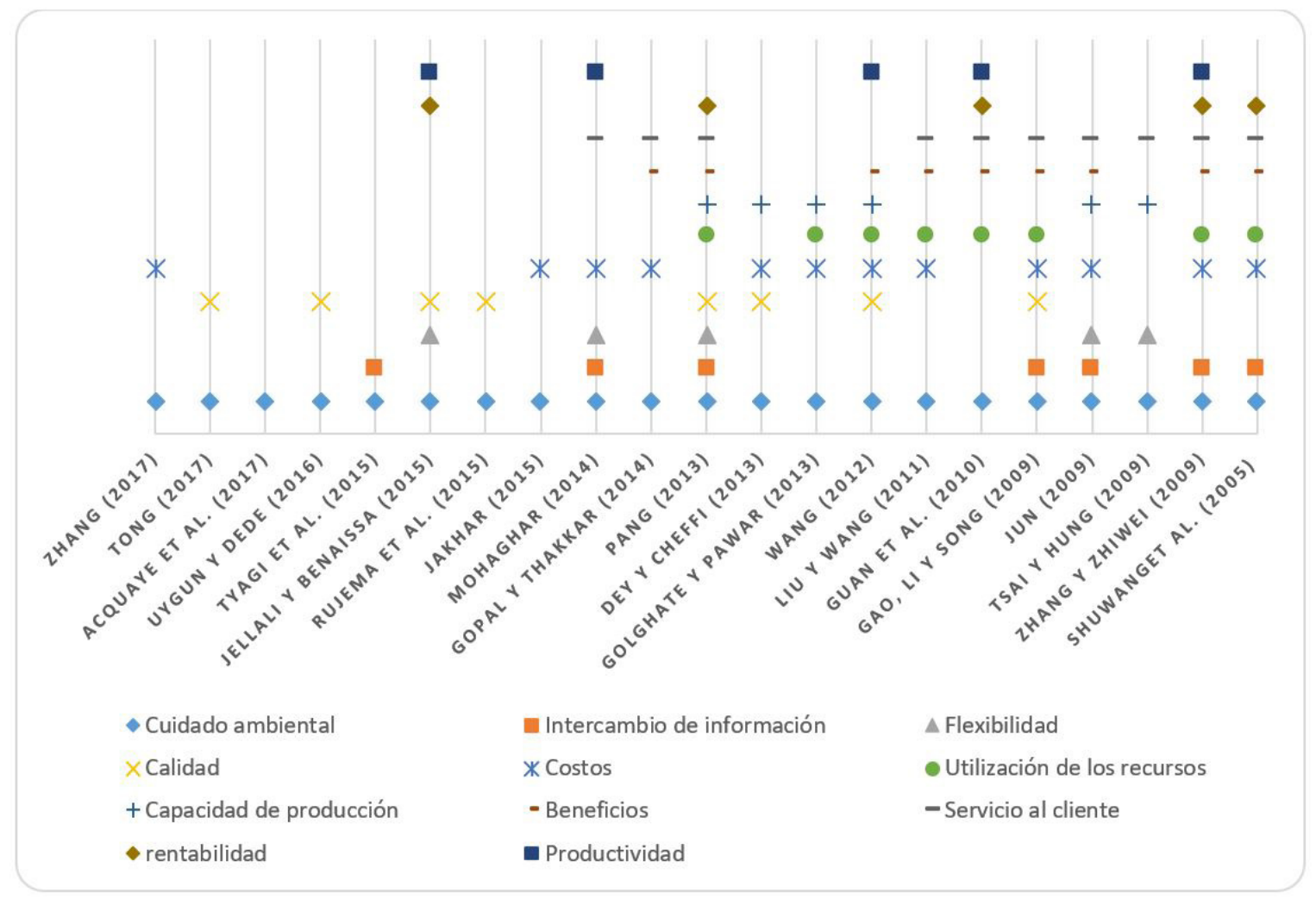

Figura 2. Criterios analizados en cada una de las investigaciones revisadas. Fuente: elaboración propia. 
- Paso 2. Establecer una escala para cada criterio. Luego de calculados e identificados los expertos, se fija la escala de evaluación para cada criterio y para el desempeño de la CSS. Para esto se emplean técnicas de recopilación de información. La aplicación de los pasos 1 y 2 será demostrada en el acápite referido a los resultados.

- Paso 3. Calcular el valor de cada criterio. Los criterios seleccionados para la evaluación del desempeño del estudio de caso, contenidos en el presente artículo, luego de aplicados los pasos anteriores, fueron servicio al cliente, productividad, rentabilidad, valor agregado e impacto ambiental. Los criterios servicio al cliente y rentabilidad se determinan de manera general para la CS, mientras que los restantes requieren un desglose por tipo de producto y proceso. La evaluación de cada uno de ellos se explica a continuación:

- Servicio al cliente. Para evaluar el nivel de servicio al cliente (NSC) en las condiciones del estudio de caso desarrollado se empleó una encuesta que fue aplicada a los clientes para analizar los aspectos, expectativas del producto, entrega de pedidos, relaciones clienteproveedor, disponibilidad del producto, calidad del producto, servicio posventa y correspondencia entre calidad y precio. Para ello, se utilizó una escala de Likert 11-pésima, 2-muy mala, 3-mala, 4-regular, 5-buena, 6-muy buena y 7-excelente). Luego se procesaron los resultados y se obtuvo el valor del NSC.

- Tasa de valor agregado. En el presente trabajo se considera la tasa de valor agregado (TVAj) como la proporción que representa el valor real que se agrega a un producto, respecto a la suma del valor que se debía agregar al mismo y el de su recuperación si resultara defectuoso en dicho proceso. Algunos de los parámetros que comprenden su determinación se refieren a la unidad de producto; y otros, a un periodo de tiempo determinado. En este último caso, se toma el año como periodo analizado. El cálculo de TVA se realiza mediante las expresiones (1), (2), (3) y (4), en las que la TVA es la tasa de valor agregado total de la CS; $V A j$, el valor real agregado al producto $j$ en unidades monetarias al año; $V A T_{j}$, el valor total que se debió agregar al producto $j$ en unidades monetarias anuales; $V A R j$ es el valor agregado total al producto $j$ reprocesados en unidades monetarias al año; $V p_{j}$ es el volumen de producción del producto $j$ en unidades de productos anuales; $V A_{i j}$, el valor agregado al producto $j$ en el proceso $i$ en unidades monetarias por unidad de producto; $C p d j$, la cantidad de productos $j$ defectuosos en unidades de productos anuales; $\operatorname{Pr}$, la cantidad total de productos $j$ recuperados en unidades de productos al año y $n$ es la cantidad de procesos de la CS. Las expresiones para el cálculo de la TVA fueron diseñadas bajo el supuesto de que todo producto defectuoso será reprocesado desde el inicio de la CS.

$$
T V A=\frac{V A_{j}}{V A T_{j}+V A R_{j}}
$$

$$
\begin{gathered}
V A T_{j}=V p_{j} * \sum_{i=1}^{n} V A_{i j} \\
V A_{j}=\left(V p_{j}-C p d_{j}\right) * \sum_{i=1}^{n} V A_{i j}+V A R_{j} \\
V A R_{j}=\operatorname{Pr}_{j} * \sum_{i=1}^{n} V A_{i j}
\end{gathered}
$$

Para calcular el VAij, se utilizó la metodología de VinajeraZamora, Marrero-Delgado y Ruiz-Morales (2017), reflejada en las expresiones (5) y (6). El término SWij expresa el peso específico promedio del proceso $i$ en el producto $j$, es decir, el nivel de importancia de un proceso en el producto que se procesa. Constituye un valor promedio de la relación de tres parámetros esenciales, que son el costo agregado por el proceso $i$ al producto $j$ en unidades monetarias por unidad de producto $\left(C_{i j}\right)$, respecto al costo total de producto para toda la CS en las mismas unidades de medida $\left(C_{j}\right)$; tiempo de elaboración del proceso i en el producto $j$ en unidades de tiempo por unidad de producto $\left(t_{i j}\right)$ respecto al tiempo del producto en la CS en iguales unidades de medida $\left(T_{j}\right)$ y, por último, el tiempo de retraso promedio total del producto $i$ en unidades de tiempo por unidad de producto $\left(D_{t i j}\right)$, respecto al tiempo total de retraso del producto en toda la CS en las mismas unidades $\left(D_{t}\right)$. De esta forma, se considera el impacto del proceso más costoso, el que más demora y el que mayores atrasos provoca. El otro término empleado es $B j$, que constituye el beneficio neto del producto $\mathrm{j}$ en unidades monetarias por unidad de producto.

$$
\begin{gathered}
S w_{i j}=\frac{1}{3}\left(\frac{t_{i j}}{T_{j}}+\frac{D t_{i j}}{D t_{j}}+\frac{C_{i j}}{C_{j}}\right) \\
V A_{i j}=S w_{i j} * B_{j}+C_{i j}
\end{gathered}
$$

- Productividad. En este criterio se propuso el análisis envolvente de datos (DEA, por sus siglas en inglés) de Charnes, Cooper y Rhodes (1978), que parte de identificar las entradas (materias primas, energía y otros insumos) y las salidas (por ejemplo productos y recursos financieros) que son analizadas para establecer pesos de esas entradas y salidas. Con estos pesos se calculan las eficiencias en el consumo de los recursos que se ubican en una matriz de eficiencia cruzada y se determina el valor promedio mediante la expresión (7), en la que $P_{j}$ es la productividad del producto $j$ en la CS y eij es la eficiencia promedio del proceso $i$ en el producto $j$. El valor de $P_{j}$ debe fluctuar entre 0 y 1 , siendo mayor la productividad en la medida en que se acerca al extremo superior. 


$$
P_{j}=\frac{1}{n} \sum_{i=1}^{n} e_{i j}
$$

- Rentabilidad. La rentabilidad debe ser calculada utilizando el modelo DUPONT de rendimiento sobre la inversión (ROI, por sus siglas en inglés) a través de la expresión (8), en la que $\mathrm{Np}$ son las ganancias netas y A los activos, ambos expresados en unidades monetarias totales.

$$
\mathrm{ROI}=\frac{N p}{A}
$$

- Impacto ambiental. Para el análisis del impacto ambiental fue utilizada la metodología del ACV. Para ello se procedió al empleo del modelo del DEA, que resultó en el procedimiento $A C V+D E A$ propuesto por Vázquez-Rowe, Iribarren, Moreira y Feijoo (2010). Sin embargo, para el presente estudio se le realizaron otras modificaciones a este procedimiento y quedó formado por los pasos siguientes: 1) obtener los datos que necesitan ser incluidos en el inventario de ciclo de vida; 2) hacer la caracterización ambiental de los procesos; y 3) calcular la ecoeficiencia de los procesos.

Las modificaciones mencionadas fueron la primera, en el tercer paso, que coincide con el tercer paso del procedimiento ACV+DEA. Se aplicó el modelo propuesto por BarbaGutiérrez, Adenso-Díaz y Lozano (2009), que toma como datos de entradas los impactos ambientales y no los recursos empleados, como el método de Vázquez-Rowe et al. (2010). La segunda modificación se deriva del enfoque en procesos utilizados, por lo que fueron incorporados los valores de $V A_{i j}$ calculados a través de la expresión (6), en lugar del precio del producto $j$ que propone el DEA. El modelo modificado se presenta mediante el conjunto de ecuaciones (9).

$$
\text { Maximizar } \gamma_{i j}=\sum_{k=1}^{c} \alpha_{k}
$$

Sujeto a:

$$
\begin{aligned}
& \sum_{i=1}^{n} I_{i k} * \lambda_{i}=I_{i k}-\alpha_{k} \\
& \sum_{i=1}^{n} V A_{i j} * \lambda_{i} \geq V A_{i j} \\
& \sum_{i=1}^{n} \lambda_{i}=1 \\
& \lambda_{i} \geq 0, \mathrm{i}=1,2, \ldots, \mathrm{n}, \alpha_{k} \geq 0, \mathrm{k}=1,2, \ldots, \mathrm{c}
\end{aligned}
$$

En este modelo (9), la variable que se debe optimizar es el valor de la ecoeficiencia del proceso $i$ en el producto $j\left(\gamma_{i j}\right)$ expresada en ecopuntos o puntos ambientales; las variables de decisión son la reducción de la categoría de impacto ambiental $K$ para llegar a la ecoeficiencia $\alpha_{k}$ en iguales unidades de medida, utilizando el Ecoindicador 99 y el vector de pesos del proceso $i\left(\lambda_{i}\right)$. Por su parte, lik es un parámetro de entrada que expresa el impacto ambiental del proceso $i$ en la categoría ambiental $k, n$ la cantidad de procesos y c cantidad de categorías de impacto ambiental.

Una vez obtenida la ecoeficiencia de cada uno de los procesos, se calcula la ecoeficiencia de la CS a través de la expresión (10), Wi es el peso promedio del proceso i según componentes ambientales (expresión 11), $n, c_{i j}$ y tij son términos ya explicados en las expresiones (3), (4) y (5), laij (T) es el impacto ambiental del proceso i al procesar el producto $j$ en un periodo de tiempo $T$ (suma de los impactos ambientales en cada una de las categorías) y thij es el tiempo normado total para el proceso i en el producto j en unidades de tiempo por unidad de producto. El resultado de la ecoeficiencia fluctúa entre 0 y 1 ; aquí son deseables aquellos valores que más se aproximen al extremo superior.

A los efectos del presente artículo, el término ecoeficiencia en la CS expresa un estado de perfección respecto a la dimensión ambiental que desde el punto de vista práctico nunca es alcanzable, por lo que siempre es posible encontrar brechas para el mejoramiento.

$$
\begin{array}{r}
\text { Ec.ef }=1-\sum_{i=1}^{n}\left[\left(1-\frac{\gamma_{i j}}{\sum_{i=1}^{n} \gamma_{i j}}\right) W_{i}\right] \\
W_{i}=0.5\left(\frac{c_{i j}^{*} t_{i j} / t n_{i j}}{\sum_{i=1}^{n}\left[c_{i j}\left(t_{i j} / t n_{i j}\right)\right]}+\frac{I a_{i j}(T)}{\sum_{i=1}^{n} I a_{i j}(T)}\right)
\end{array}
$$

- Paso 4. Determinar el peso de cada criterio. Una vez obtenido el valor de cada uno de los criterios, se calculan sus respectivos pesos. Para ello se pueden emplear métodos como el de Saaty, Entropía, Diakoulaki, Delphi y otros. En el presente artículo se aplicó el método Saaty (1980) para calcular el peso de cada indicador.

- Paso 5. Calcular SCPI. Para calcular el nivel de desempeño de la CS se deben homogenizar los criterios llevándolos todos a escalas de máximo len caso de ser necesario). Luego se determina el SCPI a través de las expresiones (12) y (13).

$$
\begin{gathered}
S C P I=1-\sum_{q=1}^{m} R p p_{q} \\
R p p_{q}=\left(M E_{q}-E_{q}\right)\left(\frac{W_{q}}{M E_{q}}\right)
\end{gathered}
$$

Donde $W_{q}$ es el peso del criterio $q, M E_{q}$ es la máxima evaluación del criterio $q, R p p q$ es la razón de puntos perdidos 
en el criterio $q, q$ es el índice de criterios, $m$ es la cantidad de criterios analizados y $E_{q}$ es la evaluación obtenida por el criterio. Si $R p p_{q}$ obtiene un valor negativo, significa que el criterio obtuvo una evaluación mejor que la evaluación máxima propuesta, por lo que los puntos perdidos en dicho criterio serán cero. Con el valor del SCPI, se evalúa el desempeño de la CS utilizando la escala propuesta por los expertos que se explica en el punto correspondiente a los resultados.

\section{Resultados}

La herramienta metodológica descrita fue aplicada a la CS de contadores de energía eléctrica (CEE) de 127 y 220 voltios (v), tomada como estudio de caso, que comprende su adquisición en el proveedor extranjero, calibración en la empresa y entrega al cliente lempresas eléctricas del país que los instalan en las viviendas). Para definir los procesos, se consideraron los dos primeros niveles propuestos por el modelo SCOR; en el primer nivel se definieron los procesos de aprovisionamiento, producción y distribución y, en el segundo, los enumerados del P1 al P10 en la figura 3 (Jamehshooran et al., 2015; Moharamkhani et al., 2017; Sellitto et al., 2015).

Los resultados de la aplicación de la herramienta metodológica se muestran a continuación:

- Paso 1. Seleccionar criterios para calcular el SCPI.

Se aplicó una encuesta a un grupo de expertos ${ }^{3}$ seleccionado previamente; estos dieron una puntuación

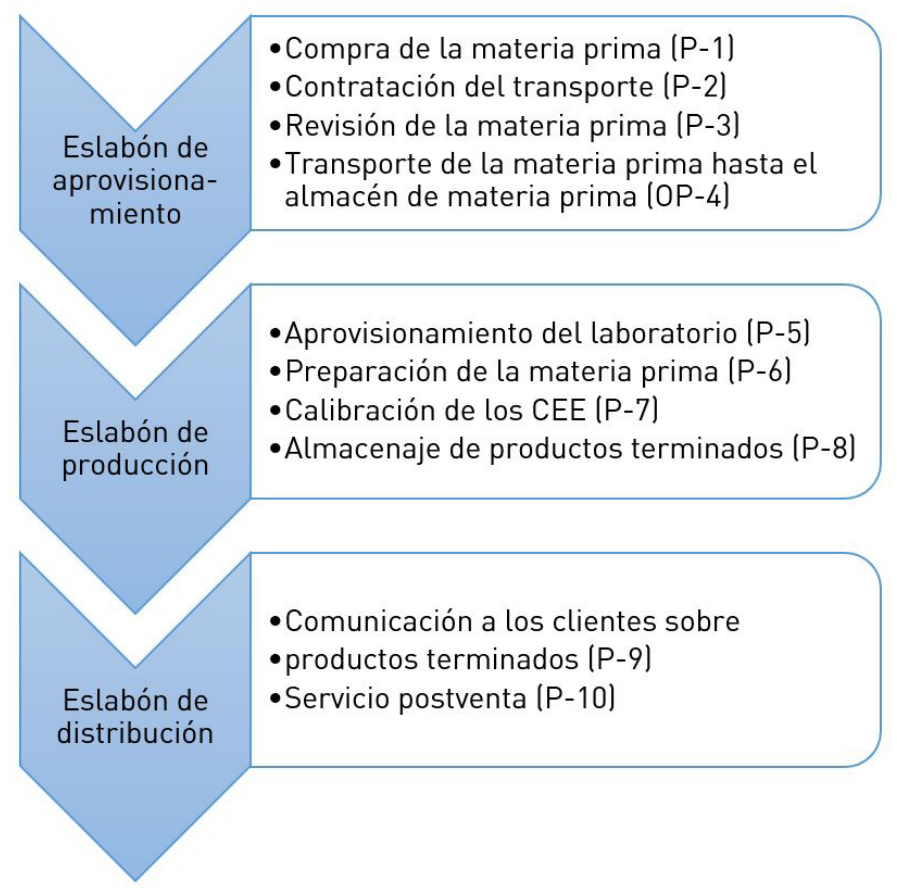

Figura 3. Procesos de la CS de CEE. Fuente: elaboración propia.

\footnotetext{
${ }^{3}$ Utilizando un método probabilístico y asumiendo una ley binomial de probabilidad, con un nivel de precisión del $8 \%(p)$, una proporción estimada de errores del $2 \%$ (i) y para un nivel de confianza del $99 \%$ (para determinar $\mathrm{k}$ ), se obtuvo una necesidad total de 9 expertos $M=\frac{p *(1-p) * k}{i^{2}}$
}

según la escala de Saaty (1980), de acuerdo con sus opiniones sobre el impacto e importancia de los criterios identificados en la literatura sobre el desempeño de la CS estudiada. Con los resultados de la encuesta, se aplicó un método de clasificación (método clúster), para seleccionar los criterios que formarían el SCPI (productividad, servicio al cliente, valor agregado, la rentabilidad e impacto ambiental). El criterio relacionado con los beneficios y las ventas no fue incluido, pues está contenido en la rentabilidad, mientras que el valor agregado fue defendido con fuerza por los expertos, a pesar de no ser uno de los recomendados en la literatura especializada (figura 2).

- Paso 2. Establecer una escala para cada criterio.

Se realizó un trabajo en equipo para determinar la escala de evaluación de cada uno de los criterios (tabla 1). Una vez determinada la cantidad de evaluaciones, se determinaron todas las posibles combinaciones para el cálculo del SCPI (5 x 5 × 5 × 4 × 5 = 2500 SCPI). Luego, fueron procesados cada uno de estos SCPI, clasificados según la escala de la tabla 1 , y se realizó un análisis discriminante para comprobar los límites de cada grupo de evaluación. Este análisis permitió corroborar la correcta clasificación del 83,4\% de los datos y crear la escala para evaluar el SCPI (última columna, tabla 1) según los resultados obtenidos del software.

- Paso 3. Calcular el valor de cada criterio.

Como parte de este paso, se calcularon los valores de cada uno de los criterios para los CEE de 127v y 220v.

- En el caso del NSC, se tomó el 100\% de los clientes encuestados. Una vez procesados los valores, se obtuvo una evaluación para este indicador de 0,93, que es evaluado como muy bueno, según su escala (tabla 1).

- Para el cálculo de la TVA se partió de la determinación del VAij a través de las expresiones (5) y (6); se obtuvieron los resultados de la tabla 2, en los que la ganancia de los CEE de $127 v$ y de $220 v$ es de 0,26 y 0,44 pesos por unidad, respectivamente.

Luego, tomando como base que el volumen total de producción fue de 137.828 contadores eléctricos (CEE) de $127 \mathrm{v}$ y 262.172 de $220 \mathrm{v}$, con 295 defectuosos (133 de 127v y 163 de 220vl, de los cuales se recuperaron 133 de $127 \mathrm{v}$ y 113 de 220v, se obtuvo una TVA de 99,87\%. Este valor es considerado como muy bueno en la tabla 1.

- Para la productividad, en la que se utilizó el modelo DEA (Charnes et al., 1978), se tomaron las entradas que se muestran en la tabla 3 , junto con el resultado de la aplicación del modelo (última columna). Luego, aplicando la expresión (7), se obtuvo un valor de 0,766, para una evaluación de bien en la tabla 1.

- La rentabilidad, al igual que el NSC, fue obtenida de manera global. Primero, se identificó el nivel de venta (7.398 900 pesos), la utilidad (162.900 pesos) y el total de activos (4.973.800 pesos). Luego, se aplicó la expresión (8), en la que se obtuvo un ROI de 0,0328 para una evaluación de regular (tabla 1). 
Tabla 1. Evaluación de cada criterio.

\begin{tabular}{lllllll}
\hline Puntos/Criterios & $\mathrm{P}$ & NSC & TVA (\%) & Ec. Ef & ROI & SCPI \\
\hline Excelente (5) & $0,95-1,00$ & $0,97-1,00$ & $99,90-100$ & & $0,13-0,15$ & $0,95-1,00$ \\
Muy buena (4) & $0,80-0,94$ & $0,9-0,96$ & $99,00-99,89$ & $0,95-1,00$ & 0,12 & $0,88-0,94$ \\
Buena (3) & $0,70-0,79$ & $0,8-0,89$ & $98,50-98,99$ & $0,85-0,94$ & $0,07-0,10$ & $0,69-0,87$ \\
Regular (2) & $0,60-0,69$ & $0,7-0,79$ & $98,00-98,49$ & $0,75-0,84$ & $0,03-0,06$ & $0,55-0,68$ \\
Mala (1) & $0-0,59$ & $0-0,69$ & $0-97,00$ & $0-0,74$ & $0-0,02$ & $0-0,54$ \\
\hline
\end{tabular}

Nota: P: Productividad; NSC: Nivel de servicio al cliente; TVA: Tasa de valor agregado; Ec.EF: Eco-eficiencia; ROI: Rentabilidad.

Fuente: elaboración propia.

Tabla 2. Valor agregado de cada proceso.

\begin{tabular}{|c|c|c|c|c|c|c|c|}
\hline \multirow[t]{2}{*}{ Proc. } & \multicolumn{2}{|c|}{$S w_{i j}$} & \multicolumn{2}{|c|}{$\begin{array}{c}\mathrm{B}_{\mathrm{j}} \\
\text { (\$/CEE) }\end{array}$} & \multirow[t]{2}{*}{$\begin{array}{c}\mathrm{CA}_{\mathrm{ij}} \\
(\$ / \mathrm{CEE})\end{array}$} & \multicolumn{2}{|c|}{$\mathrm{VA}_{\mathrm{ij}}(\$ / \mathrm{CEE})$} \\
\hline & $127 \mathrm{~V}$ & $220 v$ & $127 \mathrm{~V}$ & $220 v$ & & $127 \mathrm{~V}$ & $220 v$ \\
\hline P-1 & 0,095 & 0,098 & 0,26 & 0,44 & 0,0153 & 0,114 & 0,095 \\
\hline $\mathrm{P}-2$ & 0,025 & 0,029 & & & 0,0129 & 0,063 & 0,068 \\
\hline$P-3$ & 0,009 & 0,013 & & & 0,0003 & 0,048 & 0,049 \\
\hline$P-4$ & 0,009 & 0,013 & & & 0,0001 & 0,048 & 0,049 \\
\hline$P-5$ & 0,116 & 0,114 & & & 0,1745 & 0,239 & 0,262 \\
\hline P-6 & 0,194 & 0,186 & & & 0,3624 & 0,438 & 0,478 \\
\hline P-7 & 0,315 & 0,309 & & & 0,3124 & 0,408 & 0,472 \\
\hline P-8 & 0,196 & 0,188 & & & 0,3895 & 0,466 & 0,506 \\
\hline P-9 & 0,011 & 0,015 & & & 0,0087 & 0,057 & 0,058 \\
\hline$P-10$ & 0,031 & 0,035 & & & 0,0128 & 0,064 & 0,070 \\
\hline
\end{tabular}

Fuente: elaboración propia.

Tabla 3. Entradas a cada proceso de la CS en 8 horas de trabajo.

\begin{tabular}{lccccc}
\hline Proceso & En & $\begin{array}{c}\text { tij (horas/ } \\
\text { año) }\end{array}$ & $C_{\text {ij }}$ (\$/año) & Ob & Pr \\
\hline P-1 & 64,80 & 3,09 & 27,50 & 2 & 0,50 \\
P-2 & 40,80 & 0,51 & 23,22 & 1 & 1,00 \\
P-3 & 60,00 & 0,00 & 0,54 & 1 & 1,00 \\
P-4 & 82,40 & 0,01 & 0,13 & 2 & 1,05 \\
P-5 & 76,80 & 2,74 & 314,10 & 1 & 1,00 \\
P-6 & 121,60 & 5,30 & 652,32 & 3 & 0,33 \\
P-7 & 140,16 & 8,00 & 562,32 & 5 & 0,20 \\
P-8 & 126,40 & 5,95 & 701,10 & 2 & 0,50 \\
P-9 & 25,60 & 0,01 & 15,66 & 1 & 1,05 \\
P-10 & 15,20 & 0,73 & 23,04 & 2 & 1,07 \\
\hline
\end{tabular}

En: energía (kw-h); Ob: cantidad de obreros (u); Pr: Productividad (valor entre 0 y 11 .

Fuente: elaboración propia.

En la evaluación del impacto ambiental, se comenzó por determinar el inventario del ciclo de vida para toda la CS analizada (tabla 4).

Luego, se calcularon las categorías de impacto ambiental según el Ecoindicador 99 y se aplicó el modelo mostrado por el conjunto de ecuaciones (9), hasta obtener la ecoeficiencia para cada proceso (última columna, tabla 5). Por último, se aplicó la expresión (10), que es el resultado final de la ecoeficiencia de la CS de 0,726, para una evaluación de mal (tabla 1).
- Paso 4. Determinar el peso de cada criterio.

Para el cálculo del peso de cada criterio se aplicó el método de Saaty (1980). En la tabla 6 se muestra el valor de los criterios de la CS estudiada y los pesos calculados para cada uno de ellos. Los resultados de cada criterio se corresponden con los obtenidos en el paso 3 de la herramienta metodológica.

- Paso 5. Calcular SCPI.

Se procede con el cálculo del SCPI, que integra todos los criterios al considerar el aporte de cada uno a la sostenibilidad de la CS. La figura 4 muestra este resultado. Se puede observar para cada criterio el peso ponderado y el valor obtenido del criterio en los pasos anteriores (tabla 6), junto con su evaluación.

Aplicando la expresión (12), se obtuvo un valor de SCPI de 0,561 . Según la escala propuesta (tabla 1) el desempeño de la CS es regular. Este resultado refleja la necesidad de buscar las causas y aplicar mejoras para toda la CS. El peor comportamiento lo muestran los criterios ecoeficiencia y rentabilidad. Luego de propuestas las mejoras, se aplica de nuevo la metodología y de ser positivo su impacto se pone en práctica. En un estudio posterior realizado por VinajeraZamora, Sarache-Castro, Cespón-Castro y MarreroDelgado (2014), se detectaron las principales causas de este comportamiento y las acciones de mejora emprendidas:

- La cantidad de productos que se deben recuperar por fallas humanas y del equipo. Esto implicó el comienzo de un programa de capacitación, de revisión del sistema de mantenimiento implantado y adecuación de los puntos de control de la calidad.

Tabla 4. Inventario del ciclo de vida de cada uno de los procesos.

\begin{tabular}{lcccc}
\hline Proceso & En & In & P & D \\
\hline P-1 & 18144 & 0,86 & 0,225 & 0 \\
P-2 & 12544 & 0,79 & 0,004 & 0 \\
P-3 & 16800 & 2,86 & 0,001 & 0 \\
P-4 & 23072 & 17,14 & 0,001 & 2,6 \\
P-5 & 21504 & 2,14 & 3,82 & 0 \\
P-6 & 34048 & 2,14 & 1,72 & 0 \\
P-7 & 39245 & 2,14 & 39,2 & 0 \\
P-8 & 35392 & 2,14 & 3,85 & 0 \\
P-9 & 7168 & 0,86 & 0 & 0 \\
P-10 & P-10 & 4256 & 1,20 & 1,15 \\
\hline
\end{tabular}

En: Energía (kw-h / año); In: Infraestructura; (m²/año); P: papel (kg/año); D: Diesel (Kg/año).

Fuente: elaboración propia. 
- La existencia de reservas de capacidad tanto de equipos como de obreros, unida a dificultades de financiamiento para la compra de nuevos insumos. Como acción de mejora, se inició un estudio para el desarrollo de la CS inversa, que permitió la recuperación de los productos dañados utilizados por la población.

\subsection{Discusión de los resultados}

La herramienta metodológica y el SCPI propuestos permitieron medir el desempeño de la CS, tomada como

Tabla 5. Impacto ambiental anual de los procesos considerados.

\begin{tabular}{lcccc}
\hline Proceso & $\begin{array}{c}\text { CE } \\
\text { (eco-puntos) }\end{array}$ & $\begin{array}{c}\text { SH } \\
\text { (eco-puntos) }\end{array}$ & $\begin{array}{c}\text { R } \\
\text { (eco-puntos) }\end{array}$ & $\begin{array}{c}\text { Ec } \\
\text { (eco-puntos) }\end{array}$ \\
\hline P-1 & 11 & 34 & 18 & 0 \\
P-2 & 2 & 49 & 59 & 64 \\
P-3 & 1 & 70 & 59 & 0 \\
P-4 & 22 & 5660 & 377 & 6017 \\
P-5 & 114 & 39854 & 215 & 39999 \\
P-6 & 179 & 63079 & 315 & 63236 \\
P-7 & 208 & 72702 & 358 & 72908 \\
P-8 & 2 & 313 & 46 & 0 \\
P-9 & 0.3 & 23 & 18 & 0 \\
P-10 & P-10 & 4256 & 1,20 & 1,15 \\
\hline
\end{tabular}

CE: calidad del ecosistema; SH: Salud humana; R: recursos; Ec: eco-eficiencia.

Fuente: elaboración propia.

Tabla 6. Cálculo del peso de cada criterio.

\begin{tabular}{lccccc}
\hline $\begin{array}{l}\text { Productos/ } \\
\text { Criterios }\end{array}$ & $P$ & NSC & TVA (\%) & Ec. Ef & ROI \\
\hline $\begin{array}{l}\text { Contadores de } \\
\text { energía eléctrica }\end{array}$ & 0,766 & 0,930 & 0,998 & 0,726 & 0,0328 \\
Pesos & 0,2391 & 0,2552 & 0,1015 & 0,1934 & 0,2107 \\
\hline
\end{tabular}

Fuente: elaboración propia. objeto de estudio práctico, a partir métodos basados en múltiples criterios, con orientación a procesos y considerando las dimensiones económica, ambiental y social. Con ello, se logra llenar el vacío de conocimiento dejado por las metodologías y aplicaciones que los precedieron, planteados en la figura 1.

La CS estudiada fue evaluada como regular, según el SCPI propuesto, y los criterios de ecoeficiencia y rentabilidad fueron los principales responsables de este comportamiento. Además, fue posible medir el acierto en las acciones de mejora emprendidas, no solo en los mencionados criterios, sino sobre la sostenibilidad de toda la CSS. Los resultados mostrados son flexibles para aplicar en otras CS, con las modificaciones correspondientes, lo que garantiza su mejora continua.

Este trabajo, al igual que otros desarrollados con fines similares, tiene como limitante que, a nivel mundial, la mayor parte de las CS miden su desempeño considerando esencialmente el criterio económico, lo que dificulta realizar comparaciones en el corto plazo. Sin embargo, todo apunta a que las CSS terminarán predominando.

\section{Conclusiones}

La literatura especializada y la práctica empresarial reconocen la importancia de medir el desempeño de las CS y transitar hacia CSS. Al respecto, existen aportes importantes que constituyeron el punto de partida del presente trabajo, junto con la situación problemática definida en la CS estudiada.

Esta investigación cubre el vacío de conocimiento dejado por los autores consultados en la bibliografía especializada en la medición del desempeño de CSS, lo que facilita su monitoreo y mejoramiento continuo. Presenta como novedad la integración del enfoque en procesos, los métodos basados en múltiples criterios y la sostenibilidad. Además, el SCPI obtenido permite tomar decisiones sobre toda la CS de forma

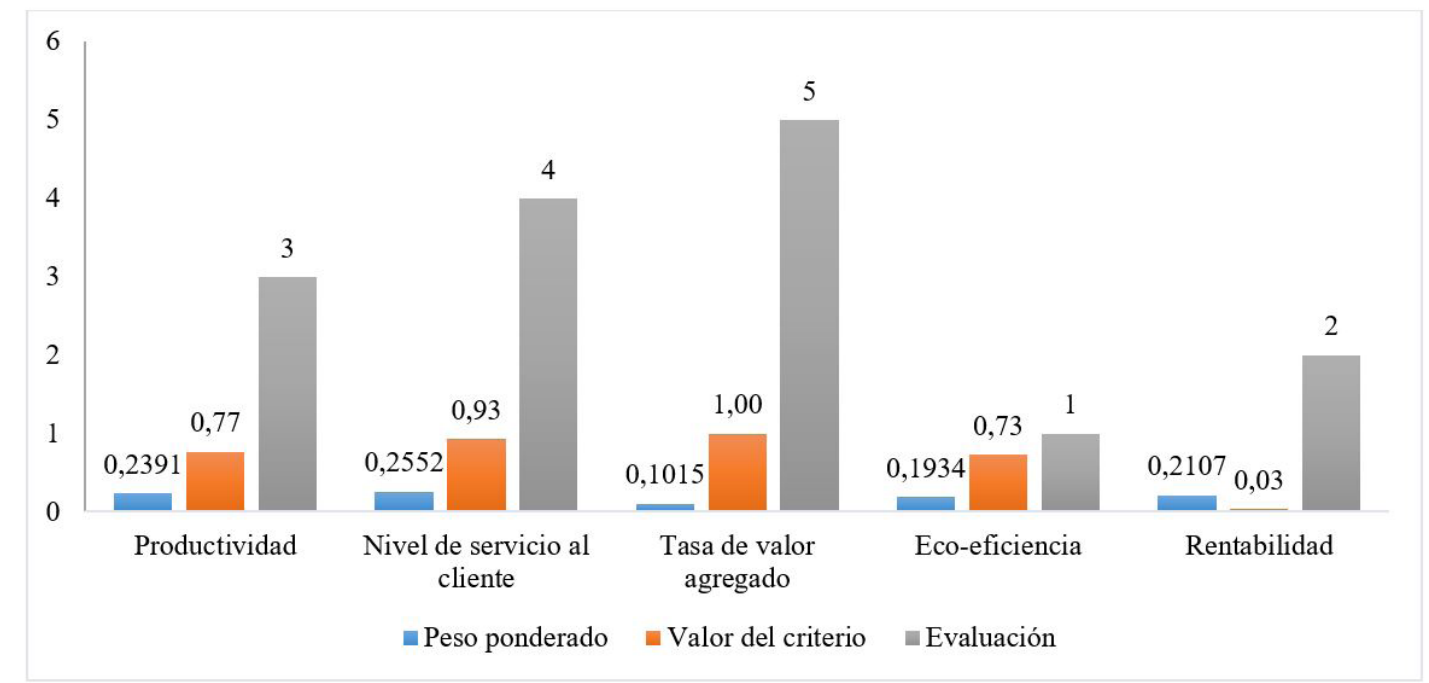

Figura 4. Datos para el cálculo del SCPI. Fuente: elaboración propia. 
integral y considerar las situaciones de conflicto que se puedan presentar.

Resultaría interesante el desarrollo de trabajos similares para evaluar la CSS con enfoque en procesos, con el empleo de otros métodos como los estadísticos y la lógica difusa, por citar solo algunos. Sin embargo, en conjunto con la necesaria generalización de trabajos como este, el mayor reto se encuentra en las investigaciones de la dimensión social de la CS, pues posee aún muchas aristas en las que se debe profundizar.

\section{Conflicto de intereses}

Los autores declaran no tener ningún conflicto de intereses.

\section{Bibliografía}

Acquaye, A., Ibn, T., Genovese, A., Afrifa, G. A., Yamoah, F. A. y Oppon, E. (2017). A quantitative model for environmentally sustainable supply chain performance measurement. European Journal of Operational Research, 268(1), 188-205. https://doi.org/10.1016/j.ejor.2017.10.057

Ahi, P. y Searcy, C. (2015). An analysis of metrics used to measure performance in green and sustainable supply chains. Journal of Cleaner Production, 86(1), 360-377. https://doi.org/10.1016/j.jclepro.2014.08.005

Ala-Harja, H. y Helo, P. (2016). Food supply chain sustainable performance in plant decision. International Journal of Advanced Logistics, 5(1), 1-18. https://doi.org/10.1080/2287108X.2015.1103542

Anand, N. y Grover, N. (2015). Measuring retail supply chain performance: Theoretical model using key performance indicators (KPIs). Benchmarking, 22(1), 135-166. https://doi.org/10.1108/BIJ-05-2012-0034

Avelar-Sosa, L., García-Alcaraz, J. L. y Maldonado-Macías, A. A. (2019). Conceptualization of supply chain performance. En Evaluation of supply chain performance: A manufacturing industry approach (pp. 69-89). Cham: Spinger. https://doi.org/10.1007/978-3-319-93876-9_5

Barba-Gutiérrez, Y., Adenso-Díaz, B. y Lozano, S. (2009). Eco-efficiency of electric and electronic appliances: A data envelopment analysis (DEA). Environmental Modeling and Assessment, 14(4), 439-447. https://doi.org/10.1007/s10666-007-9134-2

Chacón-Vargas, J. R. y Moreno-Mantilla, C. E. (2016). Organizational antecedents and capabilities for sustainable supply chain management in developing economies: The case of Colombian focal firms. Cuadernos de Administración, 29(53), 101-146. https://doi.org/10.11144/Javeriana.cao29-53.oacs

Charnes, A., Cooper, W. W. y Rhodes, E. (1978). Measuring the efficiency of decision making units. European Journal of Operational Research, 2(6), 429-444.

Chin, T. A., Tat, H. H. y Sulaiman, Z. (2015). Green supply chain management, environmental collaboration and sustainability performance. Procedia CIRP, 26, 695-699. https://doi.org/10.1016/j.procir.2014.07.035

Dey, P. K. y Cheffi, W. (2013). Green supply chain performance measurement using the analytic hierarchy process: A comparative analysis of manufacturing organisations. Production Planning and Control, 24(8-9), 702-720. https://doi.org/10.1080/09537287.2012.666859

Dossou, P. E. y Nachidi, M. (2017). Modeling supply chain performance. Procedia Manufacturing, 11, 838-845. https://doi.org/10.1016/j.promfg.2017.07.186

Egiguren, J. A. E. y Elordi, A. G. (2011). Diseño, aplicación y evaluación de un modelo para la mejora de procesos en sectores industriales maduros. Estudio del caso. DYNA, 86(1), 59-73. http://dx.doi.org/10.6036/3734

Elgazzar, S., Tipi, N. y Jones, G. (2019). Key characteristics for designing a supply chain performance measurement system. International Journal of Productivity and Performance Management, 68(2), 296-318. https://doi.org/10.1108/IJPPM-04-2018-0147

Feitó-Cespón, M., Cespón-Castro, R. y Rubio-Rodríguez, M. A. (2016). Modelos de optimización para el diseño sostenible de cadenas de suministros de reciclaje de múltiples productos. Ingeniare. Revista Chilena de Ingeniería, 24(1), 135-148.
https://doi.org/10.4067/S0718-33052016000100013

Gao, Y., Li, J. y Song, Y. (2009). Performance evaluation of green supply chain management based on membership conversion algorithm. En Proceeding: IEEE International Colloquium on Computing, Communication, Control and Management (pp. 237-240). Sanya, China: IEEE. https://doi.org/10.1109/CCCM.2009.5267895

Gey, D. G., Yusuf, Y., Menhat, M. S., Abubakar, T. y Ogbuke, N. J. (2020). Agile capabilities as necessary conditions for maximising sustainable supply chain performance: An empirical investigation. International Journal of Production Economics, 222, 107501. https://doi.org/10.1016/j.ijpe.2019.09.022

Golghate, C. D. y Pawar, M. S. (2013). Measurement and analysis of the plastic films green supply chain performance. International Journal of Intelligent Enterprise, 2(1), 21-45. https://doi.org/10.1504/IJIE.2013.057332

Gopal, P. R. C. y Thakkar, J. (2015). Development of composite sustainable supply chain performance index for the automobile industry. International Journal of Sustainable Engineering, 8(6), 366-385. https://doi.org/10.1080/19397038.2014.947392

Guan, Y. H., Cheng, H. F. y Ye, Y. (2010). Performance evaluation of sustainable supply chain based on AHP and fuzzy comprehensive evaluation. Applied Mechanics and Materials, 26-28, 1004-1007. https://doi.org/10.4028/www.scientific.net/AMM.26-28.1004

Hemalatha, S., Rao, K. N., Rambabu, G. y Venkatasubbaiah, K. (2017) Supply chain performance evaluation through AHM and Membership degree transformation. Materials Today: Proceedings, 4(8), 7848-7858. https://doi.org/10.1016/j.matpr.2017.07.120

Icarte-Ahumada, G. A. (2016). Aplicaciones de inteligencia artificial en procesos de cadenas de suministros: una revisión sistemática Applications of artificial intelligence in supply chain process: A systematic review. Ingeniare. Revista Chilena de Ingeniería, 24(4), 663679. http://dx.doi.org/10.4067/S0718-33052016000400011

Jakhar, S. K. (2015). Performance evaluation and a flow allocation decision model for a sustainable supply chain of an apparel industry. Journal of Cleaner Production, 87(15), 391-413.

https://doi.org/10.1016/j.jclepro.2014.09.089

Jamehshooran, B. G., Shaharoun, A. M. y Haron, H. N. (2015). Assessing supply chain performance through applying the SCOR Model. International Journal of Supply Chain Management, 4(1), 1-11.

Jellali, A. y Benaissa, M. (2015). Sustainable performance evaluation of the supply chain. Advanced Logistics and Transport (ICALT), 2015 4th International Conference On (pp. 151-156). Valenciennes, France. https://doi.org/10.1109/ICAdLT.2015.7136612

Jun, X. J. X. (2009). Model of Cluster Green Supply Chain Performance Evaluation Based on Circular Economy. Intelligent Computation Technology and Automation, 2009. ICICTA '09. Second International Conference On, 3, 941-944. https://doi.org/10.1109/icicta.2009.692

Kaido, B. y Katsuhito, F. (2020). Supply chain and value-added distribution of pineapple fruit in Muaro Jambi Regency, Jambi province, Indonesia. International Journal of Research in Economics and Social Sciences (IJRESS), 10(2), 50-57.

Katiyar, R., Meena, P. L., Barua, M. K., Tibrewala, R. y Kumar, G. (2018). Impact of sustainability and manufacturing practices on supply chain performance: Findings from an emerging economy. International Journal of Production Economics, 197, 303-316. https://doi.org/10.1016/j.ijpe.2017.12.007

Kumar, K. D., Shivashankar, G. S. y Kadadevaramath, R. S. (2017). Lean Supply Chain Performance Metrics for the better Manufacturing Process. Indian Journal of Science and Technology, 10(11), 1-7. https://doi.org/10.17485/ijst/2017/v10i11/106135

Kumar, V., Kumar, V., Rao, Y. y Veeramalla, S. (2019). Supply chain performance influencer in construction domain: A key factor analysis. International Journal of Supply Chain Management, 4(1), 1-7. https://doi.org/10.14419/ijet.v7i4.26.27938

Liu, X. y Wang, Z. (2011). The Performance evaluation of green supply chain of enterprise. En Q. Zhou (Ed.), Applied Economics, Business and Development (pp. 304-309). Berlin: Springer. https://doi.org/10.1007/978-3-642-23023-3_46

Mani, V., Gunasekaran, A. y Delgado, C. (2018). Enhancing supply chain performance through supplier social sustainability: An emerging economy perspective. International Journal of Production Economics, 195, 259-272. https://doi.org/10.1016/j.ijpe.2017.10.025 
Mathivathanan, D., Govindan, K. y Haq, A.N. (2017). Exploring the impact of dynamic capabilities on sustainable supply chain firm's performance using Grey-Analytical Hierarchy Process. Journal of Cleaner Production, 147, 637-653. https://doi.org/10.1016/j.jclepro.2017.01.018

Mediavilla, A., Errasti, A. y Domingo, R. (2011). Modelo para la evaluación y mejora del rol estratégico de plantas productivas. Caso de una red global de operaciones. DYNA, 86(4), 405-412.

Miri-Lavassani, K. (2018). Achieving Higher supply chain performance via business process orientation. Business Process Management Journal, 24(3), 671-694. https://doi.org/10.1108/BPMJ-07-2016-0140

Mishra, D., Gunasekaran, A., Papadopoulos, T. y Hazen, B. (2017). Green supply chain performance measures: A review and bibliometric analysis. Sustainable Production and Consumption, 10, 85-99. https://doi.org/10.1016/j.spc.2017.01.003

Mohaghar, A. (2014). Performance evaluation of green supply chain based on LFPP and balanced scorecard approach. Global Journal of Management Studies and Researches, 1(3), 158-163.

Moharamkhani, A., Amiri, A. B. y Mina, H. (2017). Supply chain performance measurement using SCOR model based on interval-valued fuzzy TOPSIS. International Journal of Logistics Systems and Management, 27(1), 115-132. https://doi.org/10.1504/IJLSM.2017.083225

Montero, M., Schmalenberg, A.-C., Quirós, O. y Doluschitz, R. (2018). Identification of supply chain performance indicators: Case study of Costa Rican coffee production. Universal Journal of Industrial and Business Management, 6(1), 1-10. https://doi.org/10.13189/ujibm.2018.060101

Narimissa, O., Kangarani-Farahani, A. y Molla-Alizadeh-Zavardehi, S. (2020). Evaluation of sustainable supply chain management performance: Dimensions and aspects. Sustainable Development, 28(1), 1-12. https://doi.org/10.1002/sd.1959

Pang, Y. (2013). Construction of green supply chain performance evaluation system based on the balanced scorecard. Advances in Information Sciences and Service Sciences, 5(8), 655-662.

Parmenter, D. (2015). Key performance indicators: Developing, implementing, and using winning KPIs. New Jersey: John Wiley \& Sons, Inc.

Rujema, S. W., Richu, S. y Aquilars, K. (2015). Effects of green purchasing strategies on sustainable supply chain performance at Unilever Tea Kenya Limited. International Journal of Innovation and Scientific Research, 15(2), 296-306.

Saaty, T. (1980). The Analytic Hierarchical Process. New York: McGraw-Hill.

Saeed, M. A. y Kersten, W. (2017). Supply chain sustainability performance indicators - a content analysis based on published standards and guidelines. Logistics Research, 10(12), 1-19. http://dx.doi.org/10.23773/2017_12

Sánchez-Flores, R. B., Ojeda-Benítez, S., Cruz-Sotelo, S. E. y NavarroGonzález, C. R. (2020). Supply chain performance improvement: A Sustainable perspective. En J. L. García-Alcaraz, C. Sánchez-Ramírez, L. Avelar-Sosa y G. Alor-Hernández (Eds.), Techniques, tools and methodologies applied to global supply chain ecosystems (pp. 333-358). Cham: Springer. https://doi.org/10.1007/978-3-030-26488-8_15

Schenk, A., Thuronyi, V. y Cui, W. (2016). Value added tax: A comparative approach. National Tax Journal, 69(1), 241-250. https://doi.org/http://dx.doi.org/10.17310/ntj.2016.1.08

Sellitto, M. A., Pereira, G. M., Borchardt, M., da Silva, R. I. y Viegas, C. V. (2015). A SCOR-based model for supply chain performance measurement: Application in the footwear industry. International Journal of Production Research, 53(16), 4917-4926. https://doi.org/10.1080/00207543.2015.1005251

Shuwang, W., Lei, Z., Zhifeng, L., Guangfu, L. y Zhang, H. (2005). Study on the performance assessment of green supply chain. 2005 IEEE International Conference on Systems, Man and Cybernetics, 1, 942-947. https://doi.org/10.1109/ICSMC.2005.1571267

Sopadang, A., Wichaisri, S. y Banomyong, R. (2017). Sustainable supply chain performance measurement a case study of the sugar industry. Proceedings of the International Conference on Industrial Engineering and Operations Management (pp. 1-12). Rabat, Morocco, april 11-13.

Sun, J., Xu, S., y Li, G. (2020). Analyzing sustainable power supply chain performance. Journal of Enterprise Information Management, ahead-ofprint. https://doi.org/10.1108/JEIM-09-2019-0296

Tong, Y. (2017). Model for evaluating the green supply chain performance under low-carbon agricultural economy environment with 2-tuple linguistic information. Journal of Intelligent and Fuzzy Systems, 32(3), 2717-2723. https://doi.org/10.3233/JIFS-16802
Tortorella, G. L., Giglio, R. y Limon-Romero, J. (2018). Supply chain performance: How lean practices efficiently drive improvements. Journal of Manufacturing Technology Management, 38(4), 934-956. https://doi.org/10.1108/JMTM-09-2017-0194

Tripathi, S., y Talukder, B. (2020). Supply chain performance and profitability in Indian automobile industry: Evidence of segmental difference. Global Business Review. https://doi.org/10.1177/0972150919898302

Tsai, W.-H. y Hung, S.-J. (2009). A fuzzy goal programming approach for green supply chain optimisation under activity-based costing and performance evaluation with a value-chain structure. International Journal of Production Research, 47(18), 4991-5017. https://doi.org/10.1080/00207540801932498

Tundys, B. y Fernando, Y. (2020). Sustainable supply chain managementKey Performance Indicators (KPI) as an element for measuring of processes. Transport Economics and Logistics, 83, 31-50. https://doi.org/10.26881/etil.2019.83.03

Tyagi, M., Kumar, P. y Kumar, D. (2015). Parametric selection of alternatives to improve performance of green supply chain management system. Procedia - Social and Behavioral Sciences, 189, 449-457. https://doi.org/10.1016/j.sbspro.2015.03.197

Tyagi, M., Kumar, P. y Kumar, D. (2017). Modelling and analysis of barriers for supply chain performance measurement system. International Journal of Operational Research, 28(3), 392-414. https://doi.org/10.1504/IJOR.2017.081912

Tyagi, M., Kumar, P. y Kumar, D. (2018). Assessment of CSR based supply chain performance system using an integrated fuzzy AHP-TOPSIS approach. International Journal of Logistics Research and Applications, 21(4), 378-406. https://doi.org/10.1080/13675567.2017.1422707

Uygun, Ö. y Dede, A. (2016). Performance evaluation of green supply chain management using integrated fuzzy multi-criteria decision making techniques. Computers \& Industrial Engineering, 102, 502-511. https://doi.org/10.1016/j.cie.2016.02.020

Vázquez-Rowe, I., Iribarren, D., Moreira, M. T. y Feijoo, G. (2010). Combined application of life cycle assessment and data envelopment analysis as a methodological approach for the assessment of fisheries. International Journal of Life Cycle Assessment, 15(3), 272-283. https://doi.org/10.1007/s11367-010-0154-9

Vinajera-Zamora, A., Sarache-Castro, W., Cespón-Castro, R. y MarreroDelgado, F. (2014). Diagnóstico del desempeño de la cadena de suministro de productos electromecánicos. Estudio de casos. IX Conferencia Internacional de Ciencias Empresariales (pp. 1-11). Santa Clara, Cuba: Editorial Feijoo.

Vinajera-Zamora, A., Marrero-Delgado, F., Coello-Machado, N., Glistau, E. (2015). Assessing the efficiency by Data Envelopment Analysis. 8th International Logistics Doctoral Student Workshop (pp. 72-91). Magdeburg, Alemania: Fraunhofer Institute.

Vinajera-Zamora, A., Marrero-Delgado, F. y Ruiz-Morales, M. (2017). Método para calcular el valor agregado en cadenas de suministro de productos electromecánico. Ingeniare. Revista Chilena de Ingeniería, 25(3), 535-546. http://dx.doi.org/10.4067/S0718-33052017000300535

Wang, F. (2012). Research on performance measurement of green supply chain management. 2nd International Conference on Economics, Trade and Development, 36, 111-114.

Zhang, H. (2017). Research on fuzzy evaluation of performance in green supply chain based on environmental economics. Journal of Intelligent \& Fuzzy Systems, 32(3), 2625-2631. https://doi.org/10.3233/JIFS-16576

Zhang, X. y Zhiwei, Z. (2009). Study of green supply chain and its performance based on fuzzy AHP and Measurement system. 2009 International Conference on E-Business and Information System Security, Wuhan (pp. 1-5). https://doi.org/10.1109/EBISS.2009.5138081 